\title{
MORTALITY IN BRAZILIAN CHILDHOOD-ONSET SYSTEMIC LUPUS ERYTHEMATOSUS PATIENTS: PREDICTORS AND CAUSES OF DEATH
}

\begin{abstract}
Ana Paula Sakamoto ${ }^{1, \star}$, Clovis A Silva², Aline G Islabão , Glaucia V Novak², Beatriz Molinari², Rosa M R Pereira², Claudia SaadMagalhães ${ }^{4}$, Amanda A Pelloso ${ }^{1}$, Claudio A Len ${ }^{1}$, Gleice Clemente ${ }^{1}$, Daniela P Piotto ${ }^{1}$, Nadia E Aikawa², Ana C Pita², Vitor C. Trindade ${ }^{2}$, Simone Appenzeller ${ }^{5}$, Virginia P Ferriani ${ }^{6}$, Marco F Silva ${ }^{7}$, Sheila K Oliveira ${ }^{8}$, Flavio R Sztajnbok ${ }^{9}$, Maria C Santos $^{10}$, Blanca E Bica8, Evaldo G Sena ${ }^{11}$, Ana J Moraes ${ }^{12}$, Melissa M Fraga ${ }^{13}$, Teresa C Robazzi ${ }^{14}$, Paulo F Spelling ${ }^{15}$, Iloite M Scheibel ${ }^{16}$, Andre S Cavalcanti ${ }^{17}$, Erica N Matos ${ }^{18}$, Luciano J Guimaraes ${ }^{19}$, Flavia P Santos ${ }^{20}$, Licia M H Mota ${ }^{19}$, Eloisa Bonfá2 , Maria T Terreri $^{1}$

1.Universidade Federal de São Paulo, São Paulo (SP), Brazil; 2.Universidade de São Paulo, São Paulo (SP), Brazil; 3.Hospital da Criança de Brasília José Alencar, Brasília (DF), Brazil; 4.Universidade Estadual Paulista “Júlio de Mesquita Filho", Botucatu (SP), Brazil; 5.Universidade de Campinas, Campinas (SP), Brazil; 6.Universidade de São Paulo, Ribeirão Preto (SP), Brazil; 7.Hospital Geral de Fortaleza, Fortaleza (CE), Brazil; 8.Universidade Federal do Rio de Janeiro, Rio de Janeiro (RJ), Brazil; 9.Universidade do Estado do Rio de Janeiro, Rio de Janeiro (RJ), Brazil; 10.Irmandade da Santa Casa de Misericórdia de São Paulo, São Paulo (SP), Brazil; 11.Universidade Federal da Paraíba, João Pessoa (PB), Brazil; 12.Universidade Federal do Pará, Belém (PA), Brazil; 13. Hospital Infantil Darcy Vargas, São Paulo (SP), Brazil; 14.Universidade Federal da Bahia, Salvador (BA), Brazil; 15.Hospital Universitário Evangélico Mackenzie, Curitiba (PR), Brazil; 16. Hospital da Criança Conceição, Porto Alegre (RS), Brazil; 17.Universidade Federal de Pernambuco, Recife (PE), Brazil; 18.Universidade Federal do Mato Grosso do Sul, Campo Grande (MS), Brazil; 19.Universidade de Brasília, Brasília (DF), Brazil; 20.Universidade Federal de Minas Gerais, Belo Horizonte (MG), Brazil.

*Corresponding author: anapaula_skmt@yahoo.com
\end{abstract}

\section{BACKGROUND}

With the introduction of new treatment strategies over the years, improvement on childhood-onset systemic lupus erythematosus (CSLE) survival is widely recognized. However, these estimates are based on isolated, short-period small studies, and information on recent trends are lacking. The objectives of the study were to identify risk factors of mortality in cSLE and to evaluate the association of these factors with clinical manifestations, laboratory features, disease activity and damage, current treatment, survival and causes of death.

\section{METHODS}

This was a multicenter, nationwide observational study of a cohort of 1,528 cSLE patients followed in 27 Brazilian pediatric rheumatology tertiary centers. Patients' charts were analyzed according to a standardized protocol at cSLE diagnosis, follow-up, last visit, or death.

\section{RESULTS}

A total of $63 / 1,528$ (4.1\%) of patients died, $53 / 63$ of them were female (84.1\%), with a median age of death of 11 years old and a median period of 3.2 years after cSLE diagnosis. Death certificates were available in 52/63 (82.5\%) patients and sepsis was the most frequent cause of death in $27 / 63$ (42.8\%) patients, followed by opportunistic infections 6/63 (9.5\%) and alveolar hemorrhage in 6/63 (9.5\%) of patients. Autopsy was performed in 12/63 (19.0\%) of the deceased cSLE patients. Regarding outcome to death, we found as determinant factors: constitutional symptoms, skin vasculitis, pericarditis, arterial hypertension, acute renal injury, chronic kidney disease (CKD), neuropsychiatric involvement (NP-SLE), thrombocytopenia, higher score of current SLEDAI-2K and SLICC/ACR-DI and higher use of cyclosporine, plasmapheresis, and intravenous immunoglobulin. In the Cox regression models, NP-SLE ( $\mathrm{HR}=2.56,95 \% \mathrm{Cl}=1.48-4.42)$ and $\mathrm{CKD}(\mathrm{HR}=4.33,95 \% \mathrm{Cl}=2.33-4.72)$ were significant risk factors associated to death. Overall patient survival after diagnosis at 5,10 and 15 years were $97.0 \%, 95.4 \%$ and $93.8 \%$ respectively.

\section{CONCLUSION}

Neuropsychiatric involvement with systemic lupus erythematosus and CKD are risk factors associated to mortality. Lupus nephritis and neuropsychiatric involvement remain major concerns in patient management. The overall mortality rate was low, most related to sepsis or infection.

\section{KEYWORDS}

Systemic lupus erythematosus, Mortality, Lupus nephritis, Neuropsychiatric, Childhood.

Realização: 